\title{
Can good broiler flock welfare prevent colonization by Campylobacter?
}

\author{
Thomas Rawson, ${ }^{*}{ }^{1}$ Frances M. Colles, ${ }^{\dagger}$ Adrian L. Smith, ${ }^{\ddagger}$ Marian Stamp Dawkins, ${ }^{\S}$ and \\ Michael B. Bonsall*
}

\begin{abstract}
*Mathematical Ecology Research Group, Department of Zoology, University of Oxford, Oxford, OX1 3PS, United Kingdom $;{ }^{\dagger}$ Peter Medawar Building for Pathogen Research, Department of Zoology, University of Oxford, South Parks Road, OX1 3SY, United Kingdom; ${ }^{\ddagger}$ Comparative Infection and Immunology Group, Peter Medawar Building for Pathogen Research, Department of Zoology, University of Oxford, South Parks Road, OX1 3SY, United Kingdom; and ${ }^{\S}$ Department of Zoology, University of Oxford, Oxford, OX2 8QJ, United Kingdom
\end{abstract}

\begin{abstract}
Using data on rearing and welfare metrics of multiple commercial broiler flocks, we investigate how welfare measures such as hock burn, mortality, and pododermatitis, among others, impact the likelihood of a flock becoming colonized by $\mathrm{Cam}$ pylobacter. Using both logistic regression and Bayesian networks, we show that, while some welfare metrics were weakly related to Campylobacter colonization, evidence could not be found to suggest that
\end{abstract}

these metrics directly exacerbated Campylobacter colonization, rather that they were both symptoms of the same parent variable - the managing company. Observed dependency on the management of the flock suggested that yet-undiscovered differences in rearing practice were the principal factor explaining both poor bird welfare and increased risk of Campylobacter, suggesting that action can be taken to improve both these factors simultaneously.

Key words: broiler, welfare, Campylobacter, logistic regression, Bayesian network

$$
\begin{array}{r}
2021 \text { Poultry Science 100:101420 } \\
\text { https://doi.org/10.1016/j.psj.2021.101420 }
\end{array}
$$

\section{INTRODUCTION}

For several years campylobacteriosis has been the most frequently observed zoonotic disease in humans throughout the EU (Westrell et al., 2009), with poultry meat identified as a leading infection route (EFSA Panel on Biological Hazards (BIOHAZ), 2011). This acute form of food poisoning, characterized by diarrhea, fever, and abdominal pain, is estimated to affect 450,000 individuals a year in the United Kingdom, approximately $10 \%$ of which result in hospitalization (Strachan and Forbes, 2010). An investigation by Public Health England into the extent of Campylobacter within the poultry industry revealed that $73 \%$ of supermarket chicken carcasses were found to contain Campylobacter and seven percent of the outer packaging was similarly contaminated (Jorgensen et al., 2015). This considerable public health burden posed by Campylobacter spp. represents an estimated $£ 50$ million annual economic cost to the UK (Tam and O'Brien, 2016).

(C) 2021 The Authors. Published by Elsevier Inc. on behalf of Poultry Science Association Inc. This is an open access article under the CC BY-NC-ND license (http://creativecommons.org/licenses/by-nc-nd/ $4.0 /)$.

Received April 7, 2021.

Accepted August 3, 2021.

${ }^{1}$ Corresponding author: t.rawson@imperial.ac.uk
Given the extent to which Campylobacter dominates commercial chicken flocks, attempting to reduce the proliferation of the pathogen at farm level would have significant impacts in reducing disease incidence in humans. Once Campylobacter is first identified within a broiler flock (chickens grown specifically for their meat), colonization of all birds occurs very rapidly (Evans and Sayers, 2000). In experimental studies, it can take only a single week for an entire flock to become colonized following the introduction of a single infected bird (Stern et al., 2001). This speed of proliferation makes identifying the initial point of entry of Campylobacter into a flock challenging, and has resulted in a focus on preventative measures.

To-date, the poultry industry has largely focused upon on-farm biosecurity measures (Fraser et al., 2010; Gibbens et al., 2001), such as boot-dips and improved cleaning of housing. However, little impact in reducing incidence has been achieved with these measures (Hermans et al., 2011). As such, research has instead turned to a broad array of preventative measures (Ghareeb et al., 2013), such as treatment of food and water (Peh et al, 2020), probiotics (Saint-Cyr et al., 2016), and bacteriophage therapy (El-Shibiny et al., 2009). Such measures have thus far had mixed, and at times contradictory, success. 
One area of research still greatly overlooked is the role of bird welfare in the emergence of Campylobacter within a flock, both as a potential indicator of Campylobacter colonization, and as a driving factor. Campylobacter spp. was long considered to be commensal within broiler chickens, but recent studies have begun to suggest they may be pathogenic under some circumstances (Humphrey et al., 2014; Wigley, 2015). Some variables have previously been observed to correlate with changes in the gut microbiota and immune response of birds, such as stocking density (Gomes et al., 2014; Guardia et al., 2011), food withdrawal, and heat stress (Burkholder et al., 2008). More directly, lesions on the footpad and arthritis have been shown to be strong predictors of Campylobacter prevalence (Alpigiani et al., 2017), further supporting findings that flock movement patterns and behavior can also accurately predict Campylobacter prevalence (Colles et al., 2016). Our own previous mathematical modeling studies have highlighted the potential for stocking density (Rawson et al., 2019) to impact the population dynamics of Campylobacter within a flock, and have also shown that the colonization status of an entire flock is greatly impacted by the most susceptible birds within the flock (Rawson et al., 2020), suggesting that attention to individual birds must not be overlooked.

This study investigates the relationship between multiple welfare indicators on Campylobacter prevalence in flocks using two different mathematical modeling approaches. We firstly employ a logistic regression analysis to test for direct relationships between Campylobacter colonization and predictor variables, such as weight, mortality, and hock burn incidence. To further investigate how our considered variables interact and influence one-another, we combine our logistic regression with a Bayesian network analysis to demonstrate the network of conditional dependencies between variables, to investigate more precisely how variables affect and impact each other. In combination with the logistic regression analysis, we are able to posit where welfare directly increases the likelihood of Campylobacter colonization, or to what extent colonization by this bacteria is a symptom of the same responsible variable.

\section{MATERIALS AND METHODS}

\section{Data}

Data was provided across 6 yr (2010 to 2015) from multiple farms throughout the United Kingdom. Each data point represents a flock of broilers, listing multiple welfare parameters and rearing information, as well as a measure of whether the flock tested positive for $\mathrm{Cam}$ pylobacter. All variables measured for flocks are detailed and defined below:

- Company-A 2-factor categorical variable, depicting whether the flock is overseen by company " 1 " or "2". This variable will also therefore capture differences in company-specific rearing methodologies not represented by our current list of predictor variables.

- Farm-A categorical variable, further delineating the Company measure, detailing which farm the flock was located at, so as to investigate trends unique to certain locations. Company 1 data contained only one farm, 'C1-F1', while company 2 data was split across 3 farms: 'C2-F1', 'C2-F2', 'C2F3'. Data from these 4 farms were further divided across $6,4,4$, and 3 separate housing sheds respectively; however, this data level was too sparse to be considered. All houses on a farm had identical house size, flock size, number of drinkers/feeders, and age of slaughter.

- Number placed-A numerical variable describing how many broilers made up the flock. While modeling studies have primarily implicated stocking density as a high Campylobacter risk factor, the total flock population may also increase the likelihood of initial flock inoculation (Rawson et al., 2019). Note that all flocks were grown to a target final stocking density of $38 \mathrm{~kg} \mathrm{~m}^{-2}$.

- Date placed-The date the flock was first placed into the house. Campylobacter is well reported to show seasonal trends, with the warmer, summer months seeing flocks test positive for Campylobacter more frequently (Djennad et al., 2019; Nylen et al., 2002).

- Breed-A 3-factor variable describing the commercial line of broilers grown. Two fast-growing commercial lines of broiler were investigated, sourced from 2 respective breeding companies, with flocks comprised of either: Breed A, Breed B, or a mixture of Breed A \& B. Host-bird genetics have been shown to impact Campylobacter prevalence (Babacan et al., 2020; Psifidi et al., 2021; Stern et al., 1990), hence the consideration of the genetic line of the flock.

- Number of parent flocks-The number of parent flocks the broiler flock was sourced from. The possibility of vertical transmission of Campylobacter from parent to chick has been primarily dismissed based upon culture methods to date (EFSA Panel on Biological Hazards (BIOHAZ), 2011), however, more sensitive genetic techniques have recently isolated Campylobacter from chicks less than 8 days old (Colles et al., 2020; Cox et al., 2012), reopening the possibility of such a transmission route. The hypothesis is that a greater number of parent flocks could increase the number of Campylobacter sequence types (and thus phenotypic specializations) that a flock is exposed to at hatch (Petersen et al., 2001).

- Mean parent age-The average age (in weeks) of all parent flocks sourced from. Parent age has been shown to impact egg weight and embryo weight (Shanawany, 1984), and thus could potentially impact the general health of the chick.

- 7/14/21/28/35/Total mortality percentage-Six different variables, describing the percentage of the flock that had died after $x$ days. 
- Pododermatitis percentage-What percentage of the flock suffered from pododermatitis; inflammation and ulcers on the footpad and toes. This was measured postmortem by abattoir staff, as the cross-sectional prevalence of any signs of footpad dermatitis, expressed as percent of that flock.

- Hock burn percentage-What percentage of the flock suffered from hock burn; areas where ammonia from the waste of other birds has burned through the skin of the leg. This was measured postmortem by abattoir staff, as the cross-sectional prevalence of any signs of hockburn, expressed as percent of that flock.

- 7/14/21/28/35/Final day weight-Six variables showing the mean weight of the flock, in grams, at weekly intervals.

- Maximum/minimum temperature-A variable describing the maximum and minimum recorded external temperature, in degrees centigrade, for the duration of time in which the flock was housed, as sourced from historical records from the Met Office for the nearest weather station.

- Campylobacter 21/28/35 days-A 2-factor variable depicting whether a flock was found to be positive or negative for Campylobacter after 21/28/35 days. This was sampled via fabric boot swabs in the flock house at $21 / 28 / 35$ days. In addition, fresh fecal samples were collected concurrently on d 28. Campylobacter prevalence was then tested for in all samples via culture methods. Full details of this methodology are given in Colles et al. (2016).

A total of 212 flocks were monitored, however not all variables could be measured for all flocks due to the practical difficulties in obtaining all measures from farms. As such there is some degree of missing data across all variables, most notably that only 149 of these flocks have a final record of Campylobacter infection status. Before incorporating this data into a mathematical model, we must consider the detail of data available given the absence of some variables for some flocks. To ensure the maximum number of flocks is used in model fitting, a balance must be found between filtering out variables to increase data availability, while not overly limiting the number of variables investigated. We detail these decisions below.

\section{Data Cleaning}

Before beginning the regression analysis, we clean and simplify our data to aid interpretation. The Campylobacter variables across time points 21,28 , and $35 \mathrm{~d}$ were simplified to a single variable that reads as positive if a flock was recorded positive on any of the 3 dates recorded, and negative if the flock was reported negative on all of the measured dates provided. This was to increase the data availability, as some flocks were only measured on certain dates. There were 6 instances of a flock being recorded as negative after previously testing positive. These 6 instances were cases where the fecal samples taken on d 28 tested positive, but the boot swab on d 35 tested negative. It was considered appropriate to rely on the more targeted fecal sample for these 6 cases. There were 8 instances whereby a flock was recorded as Campylobacter-negative on d 21 and 28, but data was not recorded for $\mathrm{d} 35$. Since it could not be assured that these flocks remained Campylobacter-negative, they were not considered for logistic regression and network fitting.

The Date placed variable was converted to a 4-level factor variable, denoting the season that the flock was reared in. This was done as date is known to have a nonlinear effect on Campylobacter prevalence (Jorgensen et al., 2011), with incidence in both flocks and humans more frequently observed in the UK summer compared to the winter (Louis et al., 2005). It is this effect that we wish to investigate as opposed to variation between years. Season classification is partitioned by the dates December 1, March 1, June 1, and September 1, aligning with the meteorological seasons, which more accurately capture temperature variation than the astronomical seasons' classification.

Regression analysis requires that the explanatory variables be independent of the response variable (and each other) otherwise predictive power is weakened across all dependent descriptor variables. In some cases, parameters of the linear model then become indeterminate due to the high degree of multicollinearity. For example, the 7/14/ 21/28/35/Total mortality percentage variables are, as expected, all highly correlated with one another; hence, we use only the 28-d mortality percentage measure, as this is the one that most data was available for. We do the same for the average bird weight variables. Likewise, the Company variable was removed for the logistic regression, as it is heavily correlated with the Farm variable (companies do not share farms), however the Farm variable was also then found to have very strong correlation with the Number placed variable. For this reason the Farm variable is also removed, as Number placed is a preferred metric of interest. Similarly, we use only the Minimum temperature, and not the Maximum temperature, or the Date placed, as these 3 are strongly correlated. By reducing the number of model predictors, the generalized variance-inflation factors (GVIF) (Fox and Monette, 1992) of all variables are less than 3, far less than the commonly-used threshold of 10 .

Finally, the data was filtered to remove any flocks with missing values for the explanatory variables under consideration. 76 data points remained for the final logistic regression model. Flocks with missing data were later utilised for the parameter learning stage of the Bayesian network model. A summary table of all variables considered in the final logistic regression model is presented in Tables 1 and 2.

\section{Logistic Regression}

Multiple logistic regression is an adaptation of multiple linear regression for instances where the response 
Table 1. Factor variable summaries.

\begin{tabular}{|c|c|c|c|c|}
\hline Variable & Factor level & Total & $\begin{array}{l}\text { Campylobacter } \\
\text { positive }\end{array}$ & $\begin{array}{c}\text { Campylobacter } \\
\text { negative }\end{array}$ \\
\hline \multirow[t]{2}{*}{ Company } & 1 & 41 & 22 & 19 \\
\hline & 2 & 32 & 17 & 15 \\
\hline \multirow[t]{4}{*}{ Farm* } & C1-F1 & 41 & 22 & 19 \\
\hline & $\mathrm{C} 2-\mathrm{F} 1$ & 15 & 7 & 8 \\
\hline & $\mathrm{C} 2-\mathrm{F} 2$ & 8 & 4 & 4 \\
\hline & C2-F3 & 9 & 6 & 3 \\
\hline \multirow[t]{3}{*}{ Breed } & $\mathrm{A}$ & 30 & 18 & 12 \\
\hline & B & 41 & 20 & 21 \\
\hline & $\mathrm{A} \& \mathrm{~B}$ & 2 & 1 & 1 \\
\hline \multirow[t]{4}{*}{ No. Parents } & 1 & 36 & 23 & 13 \\
\hline & 2 & 21 & 10 & 11 \\
\hline & 3 & 12 & 5 & 7 \\
\hline & 4 & 4 & 1 & 3 \\
\hline \multirow[t]{4}{*}{ Date placed } & Spring & 23 & 18 & 5 \\
\hline & Summer & 16 & 15 & 1 \\
\hline & Autumn & 12 & 1 & 11 \\
\hline & Winter & 22 & 5 & 17 \\
\hline
\end{tabular}

*Company 1 has only one farm 'C1-F1'. Company 2 has three farms: 'C2-F1', 'C2-F2', 'C2-F3'.

Table 2. Continuous variable summaries.

\begin{tabular}{lcc}
\hline \hline Variable & Mean & Standard deviation \\
\hline Number placed & 27,667 & 7,006 \\
Mean parent age & 38.93 & 10.07 \\
28-d mortality percentage & 3.97 & 1.41 \\
Pododermatitis percentage & 58.59 & 28.22 \\
Hock burn percentage & 21.59 & 19.66 \\
28-d average bird weight & $1,424.8$ & 83.12 \\
Minimum temperature & 6.85 & 3.71 \\
\hline
\end{tabular}

variable of interest is a 2-factor binary output $(Y \in\{0$, $1\}$ ), in our case where a flock is either Campylobacter negative or positive. A multiple linear regression model structures the response variable, $Y$, as a linear predictor of a set of explanatory variables, $X_{i}$, like so;

$Y=\beta_{0}+\beta_{1} X_{1}+\beta_{2} X_{2}+\cdots+\beta_{\mathrm{n}} \mathrm{X}_{\mathrm{n}}$

for $n$ variables, and where $\beta_{i}$ are the coefficients to be determined. A logistic regression instead models $p=$ $P(Y=1)$, the probability that $Y=1$, as:

$\operatorname{logit}(p)=\beta_{0}+\beta_{1} X_{1}+\beta_{2} X_{2}+\cdots+\beta_{\mathrm{n}} \mathrm{X}_{\mathrm{n}}$

where $\operatorname{logit}()$ is the $\log$-odds ratio $\operatorname{logit}(p)=\log \frac{p}{1-p}$, which ensures that $p$ is bounded between 0 and 1 . To model the impact of factor variables with $m$ levels, we use treatment contrasts; $m-1$ distinct descriptor variables within the model. For example, consider a simplified model which investigated the impact of breed alone on the probability of a flock being colonized by Campylobacter $(p)$. Breed has 3 factor levels; "Breed A," "Breed B," and "Breed A \& B," and therefore, the logistic regression model would be:

$\operatorname{logit}(p)=\beta_{0}+\beta_{1} X_{1}+\beta_{2} X_{2}$,

where Breed $\mathrm{A}$, is represented by $X_{1}=X_{2}=0$, Breed B by $X_{1}=1, X_{2}=0$, and the mixture of Breed A \& B by $X_{2}=1, X_{1}=0$.
Nine explanatory variables were used for the final maximal logistic regression fit: Number placed, Breed, Mean parent age, Number of parent flocks, 28-d mortality percentage, Pododermatitis percentage, Hock burn percentage, 28-d average weight, and Minimum temperature. After initially fitting the maximal model of nine explanatory variables, a step wise simplification is then performed, removing the least significant term iteratively to finally reach the minimal adequate model: a model composed of only statistically significant explanatory variables. The model was fit using the glm package in $\mathrm{R}$, which fits the model via iteratively reweighted least squares (IWLS). All code is made freely available at osf. io $/ \mathrm{pb} 62 \mathrm{~g} /$.

\section{Bayesian Network}

Bayesian networks are probabilistic graphical models that display the network of conditional dependencies between a collection of variables. Each variable in the model is visually represented as a node, with directed edges, called "arcs," between nodes representing a directly dependent relationship. $A \rightarrow B$ indicates that $B$ depends on $A$. Since arcs are directed, there is a fromand-to relationship between variables. A node with an arc directed toward another node is called a "parent" node to the respective "child" node. Each node's output is then explicitly detailed by a probability distribution that is dependent on any and all parent variables. This highlights the 2 greatest strengths of Bayesian networks as tools to investigate relationships between variables: firstly, the Markov property imposed by the network of conditional dependencies, means that the global probability distribution of the system can be expressed as a far smaller product of dependent probabilities. As such, a large and complicated probabilistic system can be simplified by knowledge of how some variables do or do not influence one another. Second, these types of models 
provide a straightforward way of visually conveying how certain explanatory variables influence (or do not influence) each other, something that would otherwise require the analysis of a large variety of logistic regression models, and could easily overlook certain dependencies. As a result of this architecture, "cycles" are by definition not allowed within a Bayesian network, meaning a path cannot be drawn from any node back to itself. Such a structure is called a directed acyclic graph (DAG). We provide a short example below to understand how such networks are calculated, but greater insight can be found in Nagarajan et al. (2013).

Calculating a Bayesian network model is separated into 2 tasks. First, structural learning: learning the network model of dependencies (i.e., identifying all arcs in the system), followed by parameter learning: finding the specific parameters of probability distributions linking parent to child nodes. Consider an example of a dataset of 3 discrete variables in a broiler flock we wish to explore: Mortality (low, average, high), Age (young, adult, old), and Feather condition (good, average, poor). We start by learning the structure between these 3 variables. Many algorithms and approaches exist for finding the structure of a Bayesian network (Bouchaala et al., 2010), however within this paper we utilize the hillclimbing algorithm (Bouckaert, 1995), a score-based structure learning algorithm. The algorithm starts with a randomly chosen graph (though usually the empty graph made up of no arcs), and calculates a network score that ascertains how effectively such a graph describes the data. It then iteratively adds, removes and reverses one arc at a time, altering the global probability distribution via the introduction (or removal) of a dependency, selecting the alteration that increases the network score the most. This process is then repeated until no further improvement can be found. Multiple network scores can be used, but we use the Bayesian information criterion (BIC) (Bhat and Kumar, 2010), a variation on the traditional likelihood function. After using this algorithm on our example data, we discover the "best" network as being the network of 2 arcs shown in Figure 1.

We see from Figure 1 that Age is a parent variable to both Mortality and Feather condition. This indicates that, from this imagined example data, Age directly informs the mortality rate of a bird and the feather condition of the bird (this result was directly demonstrated by Comin et al. (2019). An important insight gained from this network analysis would be that Mortality and

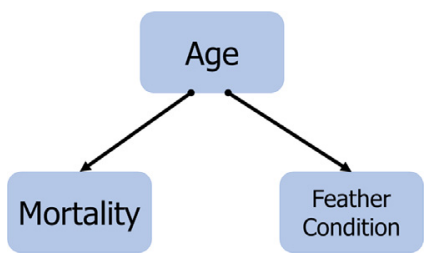

Figure 1. Bayesian network for the example problem posed. Two conditionally dependent relationships are found, from Age to Mortality and from Age to Feather condition. This example relationship was demonstrated by Comin et al. (2019).
Feather condition would likely be found to be correlated via a logistic regression analysis. However, Feather condition itself does not affect Mortality, rather they are both impacted by the same parent variable; Age. This illustrative example shows the objective of the Bayesian network approach to our particular question; what directly influences the Campylobacter status of a flock, rather than just what is correlated with Campylobacter colonization. Another advantage of such a model means that inference can be made even with missing data. The network of Figure 1 presents a structure whereby the mortality of a bird can be predicted with data on their feather condition, as this gives important indication of what the age of the bird may be. In Bayesian terms, this informs our prior belief as to the age of the bird, thus impacting our posterior belief as to the mortality of the bird. In contrast, the logistic regression approach would require an assumption on the age of such an individual, usually the mean of the training data, but no such requirement exists for Bayesian networks. Note, however, that if the age of a broiler is known, the prediction of their relative mortality rate is not improved by further information on their feather condition, as mortality is found to be predicted by age alone.

Note also from Figure 1 the mathematical advantage of such a network for expressing the joint probability distribution of the system. By definition the arcs indicate that $P$ (Age, Mortality, Feather Condition) can be expressed as

\section{$P($ Age, Mortality, Feather Condition $)$ \\ $=P($ Age $) P($ Mortality $\mid$ Age $) P($ Feather Condition $\mid$ Age $)$.}

Since each variable has 3 factor levels, this reduces a distribution of $27\left(3^{3}\right)$ parameters, to 21 , where each arc indicates that the child variable is modeled by a multinomial distribution dependent on the parent variable.

Indeed, for the second step, parameter estimation, we treat each node as being described by a multinomial distribution, and fit these using 2 separate well-known techniques, the maximum likelihood estimator (MLE), and a nested Bayesian approach, using uninformative uniform priors. See Appendix 1 for a brief introduction to Bayesian statistical inference.

A further benefit to a Bayesian network model is that we do not need to test for multicollinearity, which required us to remove several variables from consideration in the logistic regression, as structure learning specifically investigates these inter-variable correlations. As such we are able to include Company, Farm, and Date placed within our Bayesian network model. We also include the 7-d bird weight, and 7-d mortality percentage variables, alongside the 28 -d measures, to serve both as a sanity check (we would expect these 2 variables to be linked), but also to investigate the potential of age-specific effects of weight and/or mortality on Campylobacter status. This decision did however reduce the number of available structure-learning data from 76 to 73 . The 
addition of 41 flocks with low levels of missing data meant that a total of 114 flocks were used for the parameter-learning stage.

To test the significance of the fit structure, structure learning was also performed with a tabu search algorithm, and by introducing random network restarts into the hill-climbing algorithm $(10,100$, and 1,000 random restarts were all performed), all of which resulted in the same network structure. We also performed a hill-climbing structure learning algorithm using the logarithm of the Bayesian Dirichlet equivalent score (BDE) (Castelo and Siebes, 2000), as opposed to the BIC, a Bayesian-based score equivalent to the Dirichlet posterior density (and initialized with uniform priors).

When calculating conditional probabilities from the completed network structure between variables, we present results based on both a MLE and via Bayesian inference. When calculating these values via Bayesian inference, we assume uniform priors upon the values, and must define an "equivalent sample size," a parameter representing our weighting toward our initial prior assumptions. We choose a relatively low value of 10 in line with the recommendations of Scutari and Denis (2014).

All of these introduced methodologies are implemented using the bnlearn package in R (Scutari, 2009), and all code used in the model analysis is provided at osf.io/pb62g.

\section{Discretization}

While we have displayed the many inherent strength of Bayesian network models, one considerable weakness is the implementation of models consisting of both discrete and continuous variables. While methodologies exist for the assessment of such "hybrid" Bayesian networks (Scutari and Denis, 2014), the approaches are considerably more computationally demanding, and require a greater amount of data to give a robust fit to a Bayesian network. Given the comparatively smaller size of our training data $(n=73)$, we instead take the commonly used route of discretization, whereby our continuous variables are converted into discrete bins. Of the many approaches to discretization, a wide-ranging comparison by Kohavi and Sahami (1996) found the best approach to be the supervised, entropy-based minimal description length (MDL) (Fayyad and Irani, 1993) method, whereby each variable is discretized based upon its informative potential on a variable of interest. This approach was undertaken on our data, in relation to the Campylobacter variable, using the FSelectorRcpp package in R. However, only Minimum temperature was found to be able to discretized in such a way (foreshadowing our later results). As such, we instead used a quantile binning (equal-frequency) approach, to separate out our continuous variables into 3 bins of equal frequency, and confirming against the histograms for each variable that no obvious separation was missed. These bin intervals are provided in Table 3 . Three bins were
Table 3. Discretization intervals of continuous variables for the Bayesian network model.

\begin{tabular}{|c|c|c|c|}
\hline Variable & Bin 1 intervals & Bin 2 intervals & Bin 3 intervals \\
\hline $\begin{array}{l}\text { Number placed } \\
\text { (thousand chicks) }\end{array}$ & {$[11.77,22]$} & $(22,33.5]$ & $(33.5,34.65]$ \\
\hline $\begin{array}{l}\text { Mean parent age } \\
(\mathrm{wk})\end{array}$ & {$[25,31]$} & $(31,44]$ & $(44,58]$ \\
\hline $\begin{array}{l}\text { 7-d mortality } \\
\text { percentage }\end{array}$ & {$[0.7,1.25]$} & $(1.25,1.92]$ & $(1.92,7.26]$ \\
\hline $\begin{array}{l}\text { 28-d mortality } \\
\text { percentage }\end{array}$ & {$[1.98,3.18]$} & $(3.18,4.27]$ & $(4.27,9.61]$ \\
\hline $\begin{array}{l}\text { Pododermatitis } \\
\text { percentage }\end{array}$ & {$[1,48]$} & $(48,78]$ & $(78,95]$ \\
\hline $\begin{array}{l}\text { Hock burn } \\
\text { percentage }\end{array}$ & {$[0,11]$} & $(11,21]$ & $(21,90]$ \\
\hline $\begin{array}{l}\text { 7-d average bird } \\
\text { weight (grams) }\end{array}$ & {$[144,171]$} & $(171,184]$ & $(184,213]$ \\
\hline $\begin{array}{l}\text { 28-d average bird } \\
\text { weight (kilograms) }\end{array}$ & {$[1.14,1.39]$} & $(1.39,1.48]$ & $(1.48,1.57]$ \\
\hline $\begin{array}{l}\text { Minimum tempera- } \\
\text { ture (degrees } \\
\text { centigrade) }\end{array}$ & {$[1.3,4]$} & $(4,8.7]$ & $(8.7,13.8]$ \\
\hline
\end{tabular}

chosen as opposed to 4 or greater, to ensure a suitable degree of variable mixing within bins.

\section{Banned Arcs}

To both aid the structure learning process, and to disallow erroneous network structures, we also introduce a list of banned arcs, defining all arcs which are not to be considered by the algorithm, based on logical reasoning. For example, we do not allow any arcs directed toward the Company variable, as this is clearly not affected by any other variables. While the company that a flock belongs to may in turn affect the mean parent bird age for example, it is illogical to say that the mean parent bird age could affect which company the flock is managed by. Company is a variable that is predetermined before the flock even hatches, and as such cannot be influenced by factors that occur during the lifespan of the flock. A full list of these banned illogical arcs is provided with all associated code in the online repository.

\section{RESULTS}

\section{Logistic Regression}

The results of the logistic regression for the minimal adequate model are presented in Table 4, alongside a variety of model evaluation metrics. Appendix 2 shows the analysis of the original maximal model comprised of all explanatory variables, and describes the reduction steps taken to reach the minimal adequate model.

Three variables were found to be statistically significant in relation to the Campylobacter status of a flock via the Wald-test: Breed, Hock burn percentage, and Minimum temperature. Note that for the minimum adequate model, while Breed B flocks were found to be statistically significantly different to Breed A birds with relation to Campylobacter incidence, the mixed breed flocks were not found to differ from Breed A flocks. As such, the "Breed A" and "Breed A \& B" flocks were 
Table 4. Logistic regression analysis of the minimal adequate model for 84 broiler flocks using the glm function in R.

\begin{tabular}{|c|c|c|c|c|c|}
\hline Predictor & $\beta(5 / 95 \% \mathrm{CI})$ & $\mathrm{SE} \beta$ & Wald-test z-score & $P$ & $e^{\beta}$ (Odds ratio) $(5 / 95 \% \mathrm{CI})$ \\
\hline (Intercept) & $-1.085(-2.163 /-0.007)$ & 0.647 & -1.677 & 0.0935 & NA \\
\hline Breed $(1=\mathrm{B}, 0=$ Other $)$ & $-1.274(-2.328 /-0.220)$ & 0.632 & -2.015 & 0.0439 & $0.280(0.097 / 0.803)$ \\
\hline Hock burn percentage & $-0.042(-0.072 /-0.011)$ & 0.019 & -2.277 & 0.0228 & $0.959(0.931 / 0.989)$ \\
\hline Minimum temperature & $0.418(0.246 / 0.590)$ & 0.103 & 4.056 & $<<0.0001$ & $1.519(1.279 / 1.804)$ \\
\hline Test & & & $\chi^{2}$ & $P$ & \\
\hline $\begin{array}{l}\text { Overall model evaluation } \\
\text { Likelihood ratio test }^{1}\end{array}$ & & & 28.742 & $2.54 \times 10^{-6}$ & \\
\hline $\begin{array}{l}\text { Goodness of fit } \\
\text { McFadden's } R^{2} \\
\text { Cox \& Snell's } R^{2}\end{array}$ & $\begin{array}{l}0.298 \\
0.336\end{array}$ & & & & \\
\hline
\end{tabular}

Boldface indicates statistically significant $\mathrm{P}$-value $(P<0.05)$.

${ }^{1}$ Compared against null model.

collapsed into one variable for the minimal adequate model. Table 4 shows that flocks of Breed B birds were found to test positive for Campylobacter with probability 0.219 (odds ratio 0.28) compared to any other flock breed-composition. Hock burn percentage was, unintuitively, found to have a negative correlation with $\mathrm{Cam}$ pylobacter colonization. Minimum temperature was very strongly correlated, with an odds ratio showing that an increase of 1 degree to the minimum recorded temperature corresponded with a flock being 1.519 times more likely to test positive for Campylobacter. The generalized variance-inflation factors (GVIF) (Fox and Monette, 1992) of all variables in the minimal adequate model was less than 2 , and all variables of the maximal model (Appendix 2) had a GVIF of less than 3, far less than the commonly used threshold of 10 .

\section{Bayesian Network}

Figure 2 displays the final global network structure, fit using the hill-climbing algorithm, and with networks scored via BIC. The strength of individual arcs (as measured by BIC) is represented by arrow-width in Figure 2. Table 5 also explicitly provides these arc strength scores.
Table 5. Arc strengths of the Bayesian network shown in Figure 2.

\begin{tabular}{llc}
\hline \hline Parent & Child & Arc strength \\
\hline Company & Breed & -13.497 \\
Company & Farm & -43.608 \\
Company & Number placed & -38.784 \\
Company & 7-d mortality percentage & -11.506 \\
Company & Hock burn percentage & -2.551 \\
Company & 7-d weight & -4.134 \\
Company & Date placed & -1.738 \\
7-d mortality percentage & 28-d mortality percentage & -15.683 \\
7-d weight & 28-d weight & -3.660 \\
Date placed & Minimum temperature & -41.370 \\
Date placed & Campylobacter colonization & -12.976 \\
Minimum temperature & Pododermatitis percentage & -0.639 \\
\hline
\end{tabular}

Arc strength is measured by Bayesian information criterion (BIC), where a lower value indicates a stronger link.

Scoring potential networks by BDE instead of BIC resulted in a very similar optimum network structure which we present in Appendix 3. The only differences were that, 1) Pododermatitis percentage was no longer connected to any other node. 2) Minimum temperature had an additional arc from itself to Campylobacter colonization, suggesting that Campylobacter could also be impacted by temperature variation throughout the

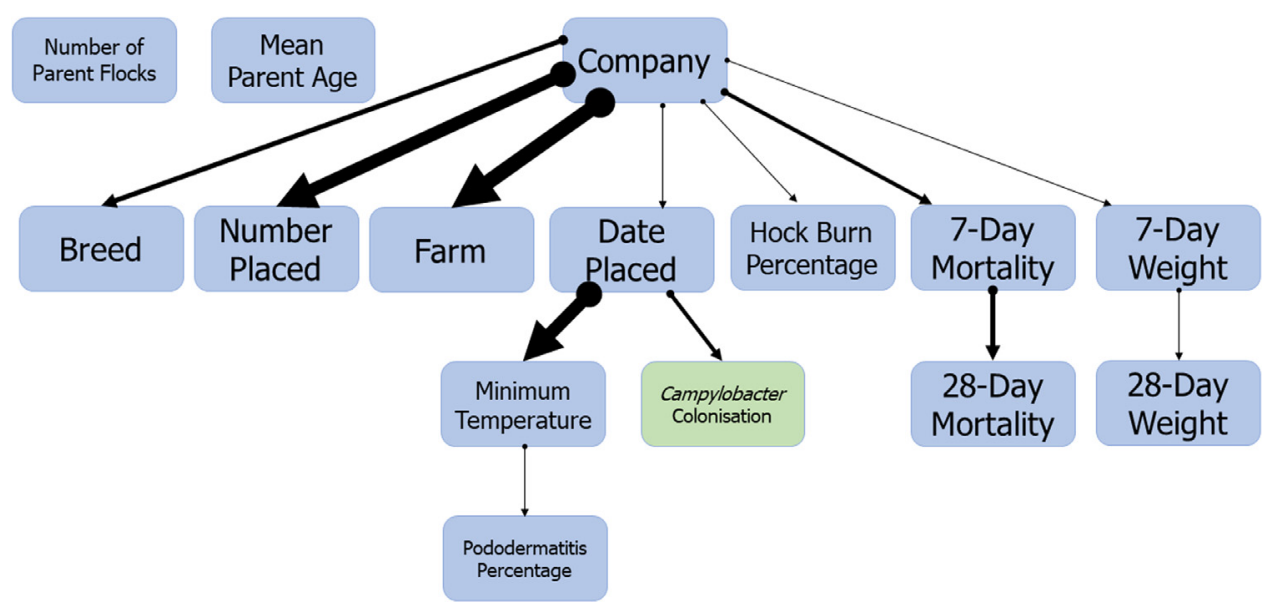

Figure 2. Bayesian network structure showing the interrelationships between multiple welfare and rearing practice factors in a flock of broilers. Campylobacter colonization is directly impacted by the season the flock is grown in. Structure was learned using a hill-climbing algorithm, and sampled networks scored using the Bayesian information criterion (BIC). Arrow-width indicates arc strength as scored by BIC, the values of which are given in Table 5 . 
season; and finally, 3) an additional arc was introduced from Date placed to Number placed, simply representing a degree of correlation between the size of flocks grown at different times of year.

Other results to be noted from Figure 2 is that neither the number of different parent flocks that a broiler flock was born from, nor the mean age of these parent was found to have any correlation to any other variable. Pododermatitis was interestingly found to be influenced by the minimum temperature recorded during the raising of the flock. We also see that many variables are directly influenced by the Company variable, suggesting that many observed differences are due to, yet unobserved, differences between management practice.

Figure 2 shows that the season (Date placed) in which a flock is reared is the sole parent node to Campylobacter status. This means that Date placed alone captures the uncertainty and probabilistic distribution of whether or not a flock is likely to test positive for Campylobacter. This means that while data on any other variable (except for the number and age of parent flocks) can inform whether or not a flock is Campylobacter positive, this data is superfluous when one has knowledge of the Date placed. The conditional probability table for Campylobacter colonization is given in Table 6 . These model parameters can be fit either via MLE or through Bayesian inference. Model parameters via both methods are provided in Table 6 . Note that one advantage of the Bayesian inference method is that this approach can learn parameters from data containing missing values. Hence while the MLE parameters are fit from the 73 data points used in structure learning, the Bayesian inference method uses 114 data points, incorporating those that were removed from structure learning due to missing values.

Conditional probability tables for Campylobacter dependent on all other variables, assuming the absence of data on any other variable, are provided in Appendix 4.

Table 6. Conditional probability tables for Campylobacter colonization status.

\begin{tabular}{lcccc}
\hline \hline \multicolumn{5}{c}{ Bayesian inference } \\
\hline \multicolumn{5}{c}{ Date placed } \\
\hline Campylobacter colonization & Spring & Summer & Autumn & Winter \\
\hline Negative & 0.273 & 0.071 & 0.878 & 0.802 \\
Positive & 0.727 & 0.929 & 0.122 & 0.198 \\
\hline
\end{tabular}

Maximum likelihood estimator

\begin{tabular}{lcccc}
\hline \multicolumn{5}{c}{ Date placed } \\
\hline Campylobacter colonization & Spring & Summer & Autumn & Winter \\
\hline Negative & 0.257 & 0.034 & 0.938 & 0.828 \\
Positive & 0.743 & 0.966 & 0.062 & 0.172 \\
\hline
\end{tabular}

We present values calculated by Bayesian inference using uniform priors, and an equivalent sample size of 10 . Below that we present values calculated via a maximum likelihood estimator.

\section{DISCUSSION}

Here, through a combination of both logistic regression and Bayesian network analysis, we have investigated the interrelationships between a selection of welfare and rearing practice explanatory variables for multiple commercial broiler flocks. At the inception of this work, our hypothesis was that poor welfare indicators such as low weight and hock burn, among others, would result in an increased risk of colonization by $\mathrm{Cam}$ pylobacter due to poor health compromising the immune response of birds in the flock (Humphrey, 2006). Social stress (Mohamed and Hanson, 1980), heat stress (Burkholder et al., 2008), and overcrowding stress (Gomes et al., 2014), have all been shown to increase susceptibility to disease in chickens by compromising the immune response (Heckert et al., 2002; Hirakawa et al., 2020), and in many cases have been correlated with increased risk of colonization with Salmonella (Alhenaky et al., 2017; Gomes et al., 2014). As such it was initially assumed that similar measures may increase incidence of Campylobacter in broiler flocks. While our work has revealed some level of correlation between poor welfare metrics and Campylobacter incidence (see the conditional probabilities of Appendix 4), these relationships were not found to be statistically significant via a logistic regression model, and our Bayesian network model suggests that poor bird welfare, as judged by the measures used here, is not in fact a cause of increased Campylobacter colonization. Despite this, our model reveals many yet-unconsidered relationships between rearing variables, provides evidence against multiple existing hypotheses, and highlights multiple promising new lines of enquiry toward identifying the source of Campylobacter colonization in commercial poultry flocks.

Our logistic regression analysis shown in Table 4, identified 3 statistically significant explanatory variables; Breed, Minimum temperature and Hock burn percentage, with $P$ values of $0.0439,5 \times 10^{-5}$, and 0.0228 , respectively. Seasonal variation in Campylobacter incidence has long been observed in broiler flocks (Jorgensen et al., 2011; Louis et al., 2005), with minimum/maximum temperature and sunshine hours significantly correlated with both the incidence and total bacterial load found in chicken flocks (Wallace et al., 1997). The warmer summer months see greater Campylobacter prevalence, yet despite the large body of research confirming this phenomenon, the precise mechanism for this increase remains unclear. While the growth rate of Campylobacter is found to vary in relation to temperature (Doyle and Roman, 1981), the minimum temperature required for Campylobacter survival is estimated to be around 30 degrees centigrade, somewhat precluding the impact of UK seasonal temperatures. Previous studies have suggested that the seasonal increase of flies (Hald et al., 2004, 2007), rodents (Meerburg and Kijlstra, 2007), and wild birds (Colles et al., 2008) as vectors of Campylobacter transmission may be responsible, while seasonal patterns in 
country-wide clonal complex incidence potentially point to genetic adaptation to seasonal trends (Jorgensen et al., 2011). Investigating this trend in human incidence of campylobacteriosis, Djennad et al. (2019) conducted a rigorous statistical assessment of spatial and weather factors, concluding that the correlation between incidence and temperature was "likely to be indirect." Our above results reach the same conclusion for broiler colonization. While our logistic regression shows the strong correlation between temperature and Campylobacter colonization, our Bayesian network analysis shows in Figure 2 that the 2 variables are conditionally independent upon the date placed, that is, the correlation is indirect.

Hock burn, the dark discoloration and ulceration of the lower leg of birds, was also found to be statistically significantly correlated with Campylobacter prevalence, however, this relationship was curiously found to be negatively correlated. These painful lesions are considered a sign of poor bird welfare, usually caused by litter unsuitably saturated with chicken waste (Allain et al., 2009). As such, the suggestion that more instances of hock burn in a flock are linked with less cases of Campylobacter is surprising, considering that the bacteria are transmitted via the fecal-oral route. One hypothesis is that the presence of Campylobacter may in turn limit colonization of the flock by more pathogenic bacteria that could more easily trigger diarrhoea within a host-bird, thus impacting the litter quality and the resulting development of hock burn. Alternatively this relationship may be an artifact of how the Hock burn percentage variable was defined. Namely it was recorded as the cross-sectional prevalence of any signs of hockburn in the flock (Dawkins et al., 2017), as opposed to the more detailed 5-point scale introduced by Michel et al. (2012), which measures both the area affected and the severity of the lesion. Our measure only captures how many birds showed signs of hock burn, and not a measure of the extremity of these burns. Bull et al. (2008) observed this same effect, whereby the flock-wide presence of hock burn was generally higher in Campylobacter negative flocks, however, the number of birds in the flock rejected from consumption due to extreme cases of hock burn was positively correlated with rates of Campylobacter colonization. Figure 2 also concludes that this correlation between Campylobacter colonization and hock burn prevalence is conditionally independent upon the managing company.

An extensive study of the influence of housing, rearing, and welfare on Campylobacter status in Icelandic poultry flocks by Seman et al. (2020) found a correlation between the amount of footpad dermatitis recorded in a flock and the overall size of the flock (Number placed), a relationship that our model was unable to identify. However, similar to our own study, of the many variables considered by Seman et al. (2020), only the season and likewise the average external temperature was found to statistically correlate with Campylobacter prevalence. They did however observe that almost all welfare indicators scored higher in Campylobacter-positive flocks.
The Bayesian network structure displayed in Figure 2 reveals a wide variety of insight into the various interrelationships of the included variables. First, we see that the number of parent flocks a broiler flock is sourced from, and the mean age of these parent flocks, had no meaningful impact on association with any other variable. The feasibility of vertical transmission of Campylobacter from parent to broiler flock is still discussed in the literature, and the inclusion of this variable was based upon the hypothesis that a greater number of parent flocks may challenge a broiler flock with a greater genotypic variety of Campylobacter isolates (Petersen et al., 2001). Parent age has also been shown to influence egg weight and embryo weight of chicks (Shanawany, 1984). Given the potential importance of maternal antibodies in suppressing Campylobacter in the first few weeks of age (Rawson et al., 2019), parent age could potentially impact the likelihood of Campylobacter colonization. Our results however indicate that factors relating to the parent flock have no effect on any of the metrics considered in this study.

The logistic regression analysis of the minimum adequate model found statistical significance in the relationship between Breed and Campylobacter colonization, where flocks of Breed A birds were more frequently observed to become colonized than Breed B birds. Caffrey et al. (2021) recently identified a correlation between breed and Campylobacter, with flocks comprised of Cobb birds, or a mixture of Cobb and Ross birds 4.75 times more likely to test positive for fluoroquinolone resistant Campylobacter jejuni than flocks comprised of just Ross birds. Further to this, Cobb birds have been found to be more frequently colonized by Campylobacter than Hubbard birds by Babacan et al. (2020), however, they were unable to separate this association from other rearing factors such as age-of-slaughter. Our Bayesian network analysis, similar to the hock burn conclusions, was unable to detect any direct arc of causation between Breed and Campylobacter colonization, suggesting that the breed of chicken is indicative of the company managing the flock, and not necessarily an indicator of a breed-specific susceptibility. Host-bird genetics have however previously been shown to cause differences in host-resistance to Campylobacter challenge (Connell et al., 2013; Li et al., 2008; Stern et al., 1990), with such resistances shown to be inheritable under experimental conditions (Boyd et al., 2005). Conventional fast-growing broiler lines, while demonstrating superior feed conversion ratios, have been shown by Abeyesinghe et al. (2021) to display poorer health outcomes regarding pododermatitis, feather coverage, mortality, and gait. Likewise, fastgrowing genetic lines have also been noted to demonstrate less "positive welfare" behaviors such as play and ground-scratching among others (Rayner et al., 2020). Further linking breed and welfare measures, Humphrey et al. (2014) found that faster-growing breeds of broiler showed evidence of prolonged inflammation in the intestines in response to Campylobacter jejuni, suggesting that the impact of breed is yet a plausible route 
of further study. Allain et al. (2009) and Martins et al. (2016) both observed that bird genotype was closely correlated with severity of footpad dermatitis, and while such a direct link was not observed in our Figure 2 network, we were able to identify an arc from Breed to Pododermatitis percentage if we relaxed our data assumptions. Above we described a data cleaning step whereby flocks that tested negative for Campylobacter on d 21 and 28, but were not measured on d 35 , were not considered within the study. If the assumption was made that such flocks were Campylobacter-negative, this allowed us to add 8 further flocks to our structure learning stage, resulting in the arc between Minimum temperature and Pododermatitis percentage being replaced by an arc from Breed to Pododermatitis percentage.

The primary conclusion of our work, as shown in Figure 2, was that our network of variables was closely related by yet-unobserved factors concealed within the Company variable. Company was found to be a parent variable to 7 factors; Breed, Farm, Number placed, Date placed, 7-d mortality, Hock burn percentage, and 7-d weight. This indicates that these seven factors significantly vary, due to which of the 2 companies considered within this study they are managed by. This suggests that choices made within the complex decision network relating to the rearing of these flocks, encompassing factors such as diet, water provision, housing, thinning protocols, cleaning regimens, antibiotic usage, and stocking density among others (Sibanda et al., 2018), will have the significant potential to both decrease incidence of Campylobacter and may simultaneously improve the welfare of the flock. While disappointing to not ascertain the primary root cause of increased Campylobacter prevalence from within our considered set of variables, the work has revealed a key network of dependencies within commonly recorded and studied metrics. While far from the first study to examine the contributions of multiple health factors toward Campylobacter colonization (Babacan et al., 2020; Frosth et al., 2020; Humphery et al., 1993; Rushton et al., 2009; Seman et al., 2020), our work is the first, to our knowledge, to utilize the powerful methodologies underlying Bayesian network analysis in studying the spread of Campylobacter. Such approaches, in combination with more traditional logistic regression analyses, greatly increase the descriptive power of gathered datasets, and it is our hope that this work will help expedite their adoption throughout the field of Campylobacter risk management. Bayesian networks have had some early success already in specifically implicating welfare measures with specific housing variables (Comin et al., 2019), we now further our attempts to identify the variables that exacerbate the spread of Campylobacter.

This study illustrates the need to investigate, more thoroughly, management decisions in the broiler industry, so as to reduce Campylobacter incidence while improving bird health and welfare, to provide the consumer with a better product whilst reducing the impact of campylobacteriosis on human health.

\section{ACKNOWLEDGMENTS}

The work was supported through an Engineering and Physical Sciences Research Council (EPSRC) (https:// epsrc.ukri.org/) Systems Biology studentship award (EP/G03706X/1) to T.R.

This work was further supported by the Biotechnology and Biological Science Research Council (BBSRC) as part of the Animal Health and Welfare ERA-net call, (grant numbers BB/N023803/1, BB/K001388) to M.S.D.

The funders had no role in study design, data collection and analysis, decision to publish, or preparation of the manuscript.

Author contributions statement: F.M.C. performed the microbiological sampling. All authors interpreted the results. T.R., M.B.B. and M.S.D. conceived the study. T.R. built the models and wrote all associated code. T.R. wrote the manuscript. M.S.D., F.M.C., and M.B.B. supervised the project. All authors reviewed the manuscript.

\section{DISCLOSURES}

The authors declare that the research was conducted in the absence of any commercial or financial relationships that could be construed as a potential conflict of interest.

\section{SUPPLEMENTARY MATERIALS}

Supplementary material associated with this article can be found in the online version at doi:10.1016/j. psj.2021.101420.

\section{REFERENCES}

Abeyesinghe, S., N. Chancellor, D. H. Moore, Y.-M. Chang, J. Pearce, T. Demmers, and C. Nicol. 2021. Associations between behaviour and health outcomes in conventional and slow-growing breeds of broiler chicken. Animal. 15:100261.

Alhenaky, A., A. Abdelqader, M. Abuajamieh, and A.-R. Al-Fataftah. 2017. The effect of heat stress on intestinal integrity and salmonella invasion in broiler birds. J. Therm. Biol. 70:9-14.

Allain, V., L. Mirabito, C. Arnould, M. Colas, S. Le Bouquin, C. Lupo, and V. Michel. 2009. Skin lesions in broiler chickens measured at the slaughterhouse: relationships between lesions and between their prevalence and rearing factors. Br. Poult. Sci. 50:407-417.

Alpigiani, I., J. C. Abrahantes, V. Michel, A. Huneau-Salaün, M. Chemaly, L. J. Keeling, A. Gervelmeyer, C. Bacci, F. Brindani, S. Bonardi, and F. Berthe. 2017. Associations between animal welfare indicators and Campylobacter spp. in broiler chickens under commercial settings: a case study. Prev. Vet. Med. 147:186-193.

Babacan, O., S. A. Harris, R. M. Pinho, A. Hedges, F. Jørgensen, and J. E. Corry. 2020. Factors affecting the species of Campylobacter colonizing chickens reared for meat. J. Appl. Microbiol. 129:10711078

Fayyad U., and K. Irani. 1993. Multi-interval discretization of continuous-valued attributes for classification learning. Accessed Aug. 2021. https://arxiv.org/abs/1309.0911.

Bouchaala, L., A. Masmoudi, F. Gargouri, and A. Rebai. 2010 Improving algorithms for structure learning in Bayesian Networks using a new implicit score. Expert Syst. Appl. 37:5470-5475.

Boyd, Y., E. G. Herbert, K. L. Marston, M. A. Jones, and P. A. Barrow. 2005. Host genes affect intestinal colonisation of 
newly hatched chickens by campylobacter jejuni. Immunogenetics. 57:248-253.

Bull, S. A., A. Thomas, T. Humphrey, J. Ellis-Iversen, A. J. Cook, R. Lovell, and F. Jorgensen. 2008. Flock health indicators and Campylobacter spp. in commercial housed broilers reared in Great Britain. Appl. Environ. Microbiol. 74:5408-5413.

Burkholder, K., K. Thompson, M. Einstein, T. Applegate, and J. Patterson. 2008. Influence of stressors on normal intestinal microbiota, intestinal morphology, and susceptibility to Salmonella enteritidis colonization in broilers. Poult. Sci. 87:1734-1741.

Caffrey, N., A. Agunos, S. Gow, K. Liljebjelke, C. L. Waldner, C. Mainali, and S. L. Checkley. 2021. A cross-sectional study of the prevalence factors associated with fluoroquinolone resistant Campylobacter jejuni in broiler flocks in Canada. Prev. Vet. Med. 186:105164.

Castelo, R., and A. Siebes. 2000. Priors on network structures. Biasing the search for Bayesian networks. Int. J. Approx. Reason. 24:39-57.

Colles, F., S. Hedges, R. Dixon, S. Preston, P. Thornhill, K. Barfod, S. Gebhardt-Henrich, P. Creach, M. Maiden, M. Dawkins. 2020. Deep sequencing reveals campylobacter in commercial meat chickens less than 8 days old. Accessed Aug. 2021. https://www.bio rxiv.org/content/10.1101/2020.06.10.141978v1.

Colles, F. M., T. A. Jones, N. D. McCarthy, S. K. Sheppard, A. J. Cody, K. E. Dingle, M. S. Dawkins, and M. C. Maiden. 2008. Campylobacter infection of broiler chickens in a free-range environment. Environ. Microbiol. 10:2042-2050.

Colles, F. M., R. J. Cain, T. Nickson, A. L. Smith, S. J. Roberts, M. C. Maiden, D. Lunn, and M. S. Dawkins. 2016. Monitoring chicken flock behaviour provides early warning of infection by human pathogen Campylobacter. Proc.e Royal Soc. B Biol. Sci. 283:20152323.

Comin, A., A. Jeremiasson, G. Kratzer, and L. Keeling. 2019. Revealing the structure of the associations between housing system, facilities, management and welfare of commercial laying hens using additive Bayesian networks. Prev. Vet. Med. 164:23-32.

Connell, S., K. G. Meade, B. Allan, A. T. Lloyd, T. Downing, C. O'Farrelly, and D. G. Bradley. 2013. Genome-wide association analysis of avian resistance to campylobacter jejuni colonization identifies risk locus spanning the cdh13 gene. G3: Genes Genomes Genet. 3:881-890.

Cox, N., L. Richardson, J. Maurer, M. Berrang, P. Fedorka-Cray, R. Buhr, J. Byrd, M. Lee, C. Hofacre, P. O'Kane, A. Lammerding, A. Clark, S. Thayer, and M. Doyle. 2012. Evidence for horizontal and vertical transmission in campylobacter passage from hen to her progeny. J. Food Prot. 75:1896-1902.

Dawkins, M., S. Roberts, R. Cain, T. Nickson, and C. Donnelly. 2017. Early warning of footpad dermatitis and hockburn in broiler chicken flocks using optical flow, bodyweight and water consumption. Vet. Rec. 180:499.

Djennad, A., G. L. Iacono, C. Sarran, C. Lane, R. Elson, C. Höser, I. R. Lake, F. J. Colón-González, S. Kovats, J. C. Semenza, T. C. Bailey, A. Kessel, L. E. Fleming, and G. L. Nichols. 2019. Seasonality and the effects of weather on Campylobacter infections. BMC Infect. Dis. 19:1-10.

Doyle, M., and D. Roman. 1981. Growth and survival of Campylobacter fetus subsp. jejuni as a function of temperature and pH. J. Food Prot. 44:596-601.

EFSA Panel on Biological Hazards (BIOHAZ). 2011. Scientific Opinion on Campylobacter in broiler meat production: control options and performance objectives and/or targets at different stages of the food chain. EFSA J. 9:2105.

El-Shibiny, A., A. Scott, A. Timms, Y. Metawea, P. Connerton, and I. Connerton. 2009. Application of a group II Campylobacter bacteriophage to reduce strains of Campylobacter jejuni and Campylobacter coli colonizing broiler chickens. J. Food Prot. 72:733-740.

Evans, S., and A. Sayers. 2000. A longitudinal study of Campylobacter infection of broiler flocks in Great Britain. Prev. Vet. Med. $46: 209-223$

Fayyad U., and K. Irani. 1993. Multi-interval discretization of continuous-valued attributes for classification learning. Accessed Aug. 2021. https://trs.jpl.nasa.gov/handle/2014/35171.

R. R. Bouckaert. 1995. Bayesian belief networks: from construction to inference. PhD Thesis. Univ. Utrecht, Utrecht, the Netherlands.

Fox, J., and G. Monette. 1992. Generalized collinearity diagnostics. J. Am. Stat. Assoc. 87:178-183.
Fraser, R. W., N. Williams, L. Powell, and A. Cook. 2010. Reducing Campylobacter and salmonella infection: two studies of the economic cost and attitude to adoption of on-farm biosecurity measures. Zoonoses Public Health. 57:e109-e115.

Frosth, S., O. Karlsson-Lindsjö, A. Niazi, L.-L. Fernström, and I. Hansson. 2020. Identification of transmission routes of Campylobacter and on-farm measures to reduce Campylobacter in chicken. Pathogens. 9:363.

Ghareeb, K., W. Awad, M. Mohnl, G. Schatzmayr, and J. Boehm. 2013. Control strategies for Campylobacter infection in poultry production. Worlds Poult. Sci. J. 69:57-76.

Gibbens, J., S. Pascoe, S. Evans, R. Davies, and A. Sayers. 2001. A trial of biosecurity as a means to control Campylobacter infection of broiler chickens. Prev. Vet. Med. 48:85-99.

Gomes, A., W. Quinteiro-Filho, A. Ribeiro, V. Ferraz-de Paula, M. Pinheiro, E. Baskeville, A. Akamine, C. Astolfi-Ferreira, A. Ferreira, and J. Palermo-Neto. 2014. Overcrowding stress decreases macrophage activity and increases Salmonella enteritidis invasion in broiler chickens. Avian Pathol. 43:82-90.

Guardia, S., B. Konsak, S. Combes, F. Levenez, L. Cauquil, J.-F. Guillot, C. Moreau-Vauzelle, M. Lessire, H. Juin, and I. Gabriel. 2011. Effects of stocking density on the growth performance and digestive microbiota of broiler chickens. Poult. Sci. 90:1878-1889.

Hald, B., H. Skovgård, D. D. Bang, K. Pedersen, J. Dybdahl, J. B. Jespersen, and M. Madsen. 2004. Flies and Campylobacter infection of broiler flocks. Emerg. Infect. Dis. 10:1490.

Hald, B., H. M. Sommer, and H. Skovgård. 2007. Use of fly screens to reduce Campylobacter spp. introduction in broiler houses. Emerg. Infect. Dis. 13:1951.

Heckert, R., I. Estevez, E. Russek-Cohen, and R. Pettit-Riley. 2002. Effects of density and perch availability on the immune status of broilers. Poult. Sci. 81:451-457.

Hermans, D., K. Van Deun, W. Messens, A. Martel, F. Van Immerseel, F. Haesebrouck, G. Rasschaert, M. Heyndrickx, and F. Pasmans. 2011. Campylobacter control in poultry by current intervention measures ineffective: urgent need for intensified fundamental research. Vet. Microbiol. 152:219-228.

Hirakawa, R., S. Nurjanah, K. Furukawa, A. Murai, M. Kikusato, T. Nochi, and M. Toyomizu. 2020. Heat stress causes immune abnormalities via massive damage to effect proliferation and differentiation of lymphocytes in broiler chickens. Front. Vet. Sci. 7:46.

Humphery, T., A. Henley, and D. Lanning. 1993. The colonization of broiler chickens with Campylobacter jejuni: some epidemiological investigations. Epidemiol. Infect. 110:601-607.

Humphrey, S., G. Chaloner, K. Kemmett, N. Davidson, N. Williams, A. Kipar, T. Humphrey, and P. Wigley. 2014. Campylobacter jejuni is not merely a commensal in commercial broiler chickens and affects bird welfare. MBio. 5:e01364-14.

Humphrey, T. 2006. Are happy chickens safer chickens? Poultry welfare and disease susceptibility. Br. Poult. Sci. 47:379-391.

Jorgensen, F., J. Ellis-Iversen, S. Rushton, S. Bull, S. Harris, S. Bryan, A. Gonzalez, and T. Humphrey. 2011. Influence of season and geography on Campylobacter jejuni and C. coli subtypes in housed broiler flocks reared in Great Britain. Appl. Environ. Microbiol. 77:3741-3748.

Jorgensen, F., R. H. Madden, E. Arnold, A. Charlett, and N. C. Elviss. 2015. FSA poject FS241044 - survey report - a microbiological survey of Campylobacter contamination in fresh whole UK produced chilled chickens at retail sale (2014-15). Accessed Aug 2021. https://www. food.gov.uk/sites/default/files/media/document/Final\%20Report\% 20for\%20FS241044\%20Campylobacter\%20Retail\%20survey.pdf.

Kohavi R., and M. Sahami. 1996. Error-based and entropy-based discretization of continuous features. KDD-96 Proceedings of the Second International Conference on Knowledge Discovery and Data Mining, Portland, OR.

Li, X., C. Swaggerty, M. Kogut, H. Chiang, Y. Wang, K. Genovese, H. He, N. Stern, I. Pevzner, and H. Zhou. 2008. The paternal effect of campylobacter jejuni colonization in ceca in broilers. Poult. Sci. $87: 1742-1747$

Louis, V. R., I. A. Gillespie, S. J. O'Brien, E. Russek-Cohen, A. D. Pearson, and R. R. Colwell. 2005. Temperature-driven Campylobacter seasonality in England and Wales. Appl. Environ. Microbiol. 71:85-92. 
Martins, B., M. Martins, A. Mendes, B. Fernandes, and E. Aguiar. 2016. Footpad dermatitis in broilers: differences between strains and gender. Braz. J. Poult. Sci. 18:461-466.

Meerburg, B. G., and A. Kijlstra. 2007. Role of rodents in transmission of Salmonella and Campylobacter. J. Sci. Food Agric. 87:2774-2781.

Michel, V., E. Prampart, L. Mirabito, V. Allain, C. Arnould, D. Huonnic, S. Le Bouquin, and O. Albaric. 2012. Histologicallyvalidated footpad dermatitis scoring system for use in chicken processing plants. Br. Poult. Sci. 53:275-281.

Mohamed, M. A., and R. Hanson. 1980. Effect of social stress on Newcastle disease virus (LaSota) infection. Avian Dis. 908-915.

Nagarajan, R., M. Scutari, and S. Lèbre. 2013. Pages 125-127 in Bayesian Networks in R. Springer. New York, NY.

Nylen, G., F. Dunstan, S. Palmer, Y. Andersson, F. Bager, J. Cowden, G. Feierl, Y. Galloway, G. Kapperud, F. Megraud, K. Molbak, L. R. Petersen, and P. Ruutu. 2002. The seasonal distribution of campylobacter infection in nine european countries and new zealand. Epidemiol. Infect. 128:383-390.

Peh, E., S. Kittler, F. Reich, and C. Kehrenberg. 2020. Antimicrobial activity of organic acids against Campylobacter spp. and development of combinations-a synergistic effect?. PLoS One 15: e0239312.

Petersen, L., E. Nielsen, and S. L. On. 2001. Serotype and genotype diversity and hatchery transmission of Campylobacter jejuni in commercial poultry flocks. Vet. Microbiol. 82:141-154.

Psifidi, A., A. Kranis, L. M. Rothwell, A. Bremner, K. Russell, D. Robledo, S. J. Bush, M. Fife, P. M. Hocking, G. Banos, D. A. Hume, J. Kaufman, R. A. Bailey, S. Avendano, K. A. Watson, P. Kaiser, and M. P. Stevens. 2021. Quantitative trait loci and transcriptome signatures associated with avian heritable resistance to campylobacter. Sci. Rep. 11:1-18.

Rawson, T., M. S. Dawkins, and M. B. Bonsall. 2019. A mathematical model of campylobacter dynamics within a broiler flock. Front. Microbiol. 10:1940.

Rawson, T., R. S. Paton, F. M. Colles, M. C. Maiden, M. S. Dawkins, and M. B. Bonsall. 2020. A mathematical modeling approach to uncover factors influencing the spread of campylobacter in a flock of broiler-breeder chickens. Front. Microbiol. 11:2481.

Rayner, A. C., R. C. Newberry, J. Vas, and S. Mullan. 2020. Slowgrowing broilers are healthier and express more behavioural indicators of positive welfare. Sci. Rep. 10:1-14.
Rushton, S., T. Humphrey, M. Shirley, S. Bull, and F. Jørgensen. 2009. Campylobacter in housed broiler chickens: a longitudinal study of risk factors. Epidemiol. Infect. 13:1099-1110.

Saint-Cyr, M. J., M. Guyard-Nicodème, S. Messaoudi, M. Chemaly, J.-M. Cappelier, X. Dousset, and N. Haddad. 2016. Recent advances in screening of anti-Campylobacter activity in probiotics for use in poultry. Front. Microbiol. 7:553.

Scutari, M. 2009. Learning Bayesian networks with the bnlearn R package. Accessed Aug. 2021. https://www.arxiv.org/abs/ 0908.3817v2.

Scutari, M., and J.-B. Denis. 2014. Bayesian Networks: With Examples in R. CRC press, Oxfordshire, UK.

Seman, M., G. Gregova, and P. Korim. 2020. Comparison of campylobacter spp. and flock health indicators of broilers in iceland. Ann. Agr. Environ. Med.: AAEM. 27:579-584.

Shanawany, M. 1984. Inter-relationship between egg weight, parental age and embryonic development. Br. Poult. Sci. 25:449-455.

Sibanda, N., A. McKenna, A. Richmond, S. C. Ricke, T. Callaway, A. C. Stratakos, O. Gundogdu, and N. Corcionivoschi. 2018. A review of the effect of management practices on campylobacter prevalence in poultry farms. Front. Microbiol. 9:2002.

Stern, N. J., R. J. Meinersmann, N. A. Cox, J. S. Bailey, and L. C. Blankenship. 1990. Influence of host lineage on cecal colonization by campylobacter jejuni in chickens. Avian Dis. 602-606.

Stern, N. J., N. A. Cox, M. T. Musgrove, and C. Park. 2001. Incidence and levels of Campylobacter in broilers after exposure to an inoculated seeder bird. J. Appl. Poult. Res. 10:315-318.

Strachan, N. J., and K. J. Forbes. 2010. The growing UK epidemic of human campylobacteriosis. Lancet North Am. Ed. 37:665-667.

Tam, C. C., and S. J. O'Brien. 2016. Economic cost of Campylobacter, norovirus and rotavirus disease in the United Kingdom. PLoS One. 11:e0138526.

Wallace, J., K. Stanley, J. Currie, P. Diggle, and K. Jones. 1997. Seasonality of thermophilic Campylobacter populations in chickens. J. Appl. Microbiol. 82:219-224.

Westrell, T., N. Ciampa, F. Boelaert, B. Helwigh, H. Korsgaard, M. Chríel, A. Ammon, and P. Mäkelä. 2009. Zoonotic infections in Europe in 2007: a summary of the EFSA-ECDC annual report. Eurosurveillance. 14:19100.

Wigley, P. 2015. Blurred lines: pathogens, commensals, and the healthy gut. Front. Vet. Sci. 2:40. 